\title{
IMPORTANCIA DEL FACTOR COLABORACIÓN DENTRO DE UNA COMUNIDAD VIRTUAL EN LA ENSEÑANZA DEL DERECHO
}

\section{IMPORTANCE OF THE FACTOR COLLABORATION WITHIN A VIRTUAL COMMUNITY IN THE EDUCATION OF LAW}

\author{
Franklin Steve Salazar Visurraga \\ Maestro en Ingeniería de Computación y Sistemas con mención en Gestión de TI \\ Universidad de San Martín de Porres \\ ORCID: 0000-0002-5701-0467 \\ fsalazarv@usmp.pe \\ Perú
}

\section{SUMARIO}

- Introducción

- Concepción de una educación a distancia

- Sistemas tradicionales y contemporáneos para soportar la educación a distancia

- Importancia del diseño de un entorno virtual centrado en el estudiante

- Consideración de la educación virtual como alternativa en la enseñanza del derecho

- El sentido de comunidad basada en la colaboración dentro de la enseñanza virtual de la rama del derecho

- Metodología

- Análisis de los resultados y prueba de hipótesis

- Discusión

- Conclusiones

- Fuentes de información

\section{ÍNDICE DE TABLAS}

- Tabla $\mathrm{N}^{\circ}$ 1: Estadísticas Descriptivas de la Dimensión Comunidad

- Tabla $\mathrm{N}^{\circ}$ 2: Grupo de Muestras y Dimensión Comunidad

- Tabla $N^{\circ}$ 3: Resultado de la Prueba de Chi-Cuadrado

\section{ÍNDICE DE FIGURAS}

- Figura $\mathrm{N}^{\circ}$ 1: Componentes del sistema de educación a distancia según el modelo de Lorenzo García Aretio

- Figura $\mathrm{N}^{\circ}$ 2: Comparación de Sistemas Tradicionales y Sistemas Contemporáneos

- Figura $\mathrm{N}^{\circ}$ 3: Relación entre Foro de Discusión y la Dimensión Comunidad

- Figura $\mathrm{N}^{\circ}$ 4: Relación entre Comunicación Fluida y la Dimensión Comunidad

- Figura $\mathrm{N}^{\circ}$ 5: Relación entre Colaboración de Compañeros y la Dimensión Comunidad

- Figura $\mathrm{N}^{\circ}$ 6: Dimensión Comunidad basada en la colaboración

\section{RESUMEN}

La investigación tuvo por objeto medir el factor colaboración dentro de un entorno virtual de una rama del derecho $y$ determinar cuánto ha evolucionado con respecto a otro entorno analizado por una tesis de investigación de la misma rama jurídica, la cual tomó una muestra con las mismas características a la muestra tomada por la investigación desarrollada por el 
suscrito. La investigación rescata la diferencia de dos tipos de sistemas educativos virtuales: tradicionales $y$ contemporáneos. Los sistemas educativos tradicionales hacen referencia a aquellas plataformas que sostienen un escenario virtual con una estructura más rígida y una comunidad más limitada. Por otro lado, los sistemas educativos contemporáneos hacen referencia a aquellas plataformas que sostienen un escenario virtual con una estructura más flexible y que alberga una comunidad más amplia. La tesis de investigación que se tomó como referencia concluyó que el nivel del factor colaboración en los sistemas educativos virtuales tradicionales era muy bajo por un débil acompañamiento de los compañeros y poca interacción en el escenario. Sin embargo, la muestra que analizó el articulo determinó que el factor colaboración tuvo un mayor nivel que la muestra analizada por la tesis, debido a que surgieron mejoras en el camino que se relacionaron con las recomendaciones brindadas por dicha investigación de referencia. Por tal motivo, la presente investigación rescata como esencial que una comunidad virtual esté basada en la colaboración para el beneficio del estudiante, generando con ello menos rechazo en el proceso de enseñanza aprendizaje.

\section{ABSTRACT}

The research tried to measure the collaborative factor within a virtual environment of the branch of law and determine how much has evolved with respect to another environment analyzed by a research thesis of the same branch, which took a sample with the same characteristics to the sample taken by this article. The research rescues the difference between two types of virtual educational systems: traditional and contemporary. Traditional education systems refer to those platforms that support a virtual scenario with a more rigid structure and a more limited community. On the other hand, contemporary education systems refer to those platforms that support a virtual scenario with a more flexible structure and that houses a wider community. The research thesis that was taken as a reference concluded that the level of the collaboration factor in traditional virtual education systems was very low due to weak companion accompaniment and little interaction on the stage. However, the sample that analyzed the article determined that the collaboration factor had a higher level than the sample analyzed by the thesis, due to improvements in the path that were related to the recommendations provided by said reference research. For this reason, the article rescues as essential that a virtual community is based on collaboration for the benefit of the student, thereby generating less rejection by the branch of law in the teaching-learning process.

\section{PALABRAS CLAVES}

Comunidad; colaboración; escenario virtual; derecho; estudiante; docente; enseñanza y aprendizaje.

\section{KEYWORDS}

Community, collaboration; virtual stage; law; student; teacher; teaching and learning.

\section{INTRODUCCIÓN}

Nuestra sociedad vive en constante interacción en muchas áreas y como seres sociables para construir conceptos e ideas nuevas necesitamos de otros miembros de nuestro circulo para lograrlo.

Existen muchas maneras de interactuar presencialmente; los juegos o métodos flexibles para motivar a la persona desde su crecimiento son cada vez más constantes en su aplicación. No es un secreto que desde que el ser humano está en un ámbito de educación inicial se le intenta ayudar a 
desarrollar destrezas o a adquirir conocimientos a través de juegos o métodos más recreativos.

Algunos factores externos o internos podrían limitar o determinar que tanto el ser humano puede relacionarse, pero no cabe duda de que el estar en una comunidad favorece al desarrollo de aprendizaje. Este aprendizaje oscila desde costumbres hasta conceptos muy elaborados.

Debido a los cambios constantes que se suscitan en la vida cotidiana se producen necesidades nuevas; y ello no es ajeno al mundo de la educación a distancia o educación virtual, ya que se traduce en incorporación de estrategias, metodologías de estudio y aprendizaje durante el tiempo, de las cuales se destacan las tecnologías de la información y de la comunicación (TIC), que soportan todas las innovaciones y ofrecen grandes ventajas para las personas que disponen de tiempo en horas no convencionales o que tienen algún inconveniente (García, 2006), tratando de reducir de esta manera brechas de tiempo y distancia.

Se destaca distintas formas que contribuyeron a implementar una educación a distancia a lo largo de la historia, y ello dependió de lo que se creía prioritario atender para establecer el soporte adecuado. Poco a poco las necesidades y las ganas de romper barreras fueron evolucionando; así como también los mecanismos y acciones para conseguirlo. Según Florido Bacallao, R. y Florido Bacallao, M. (2003):

Pese a que desde sus inicios este tipo de enseñanza tuvo que enfrentarse a la desconfianza de quienes veían en ésta una oportunidad menor, o quienes temían el desarrollo de un sistema más flexible, más dinámico y por supuesto más atractivo, no cabe duda que existía un creciente interés por la educación a distancia. (Pág. 3)

La educación virtual ha ido incursionando con más fuerza en estos últimos tiempos como una alternativa en el proceso de aprendizaje de distintas áreas, que lejos de querer reemplazar a la enseñanza presencial, no solo busca apoyar al estudiante a acceder a contenidos que físicamente no podrían, sino también a mejorar su capacidad mediante escenarios que propicien una interacción para la construcción de conocimiento. Para estos escenarios se fueron desarrollando una serie plataformas en el tiempo, que de manera evolutiva fue soportando mejor la comunicación en los programas educativos virtuales.

La finalidad de las plataformas virtuales es la de brindar un escenario centrado en el estudiante, aunque muchas veces no se ha dado así. Como se mencionó anteriormente estas tecnologías fueron creciendo para poder otorgar un espacio más idóneo al estudiante. A pesar de ello, algunas plataformas seguían manteniendo poca interacción en sus escenarios y las investigaciones fueron tomando distintos lados de interés para encontrar las posibles causas a este comportamiento.

Sin lugar a duda las tecnologías representan un factor clave para propiciar una flexibilidad mediante los recursos que albergan, pero ello debe sumarse a la capacidad de guiar un trabajo colaborativo, aspecto que cada vez cobra más presencia e importancia en las propuestas educativas virtuales y más aún en coyunturas difíciles.

De acuerdo con Ossiannilson (2012), los escenarios virtuales deben conservar un ambiente flexible para el desarrollo de las actividades. La búsqueda constante del mejor escenario debe ser la prioridad de las instituciones que desean desarrollar programas académicos o de capacitación.

Dentro de la búsqueda constante de calidad se han dado estudios que invitan a la reflexión para entender mejor como se han ido perfilando las mejoras de los ambientes virtuales de aprendizaje desde su 
comprensión de algunos componentes dentro del proceso de enseñanza y aprendizaje. Dentro de este proceso se encuentra el estudiante o también mencionado en estudios realizados como el usuario final.

Si hablamos de calidad en un entorno virtual educativo para el estudiante necesariamente debe resaltarse el papel del internet porque con su aparición se pudo extender alternativas para soportar e impulsar diversos procesos de la sociedad, entre ellos la educación, surgiendo progresivamente sistemas que apostaron por fortalecer la creación de comunidades de aprendizaje. Las condiciones con que la educación a distancia fue abordada, dependieron de las políticas que las instituciones creían conveniente sostener en el tiempo como se acotó previamente. Se pudo observar comunidades pequeñas hasta comunidades muy grandes que involucraban participantes de distintas localidades. Los sistemas educativos virtuales al margen del tamaño de su comunidad deben procurar crear un sentimiento de acompañamiento basado en la colaboración.

El nivel colaborativo en los sistemas educativos virtuales resulta de mucha importancia para el presente artículo, el cual establece que el más beneficiado en toda innovación tecnológica en la educación debe ser el estudiante y con mayor razón aun en la rama del derecho, donde la interacción constante e intercambio de experiencias es la base de formación y construcción de conocimiento.

\section{CONCEPCIÓN DE UNA EDUCACIÓN A DISTANCIA}

La implementación de una educación a distancia tuvo que lidiar con más de un inconveniente hasta la actualidad, entre ellos la consideración de la misma para poder enfrentar el desafío de lograr que el estudiante tenga un aprendizaje integro en un escenario diferente. La educación a distancia no siempre fue vista como una alternativa para la construcción de conocimiento, pero poco a poco la sociedad se fue adaptando a los beneficios que esta modalidad tiene.

A través de los años, diversos aportes intentaron moldear la conceptualización de esta innovación en el ámbito educativo para su mejor entendimiento y reconocer con ello su importancia frente a las adversidades por acceder a una formación superior en las distintas ramas educativas. La construcción de conocimiento siempre fue una necesidad en distintos sectores y la inserción de una nueva modalidad basada en una conexión síncrona y asíncrona fue progresivamente paliando esta dolencia en el campo del aprendizaje.

García Aretio (2002) en su estudio se refiere a la educación a distancia como: "La construcción científica que consiste en la sistematización de las leyes, ideas, principios y normas, con objeto de describir, explicar, comprender y predecir el fenómeno educativo en la modalidad a distancia y regular la intervención pedagógica en este ámbito" (Pág. 79).

Resulta importante destacar que la regulación de todas las partes que envuelve esta modalidad es vital para transmitir transparencia en los programas de las instituciones educativas. Es muy probable que la regulación del trabajo educativo haya sido un factor clave para desconfiar en esta modalidad durante un tiempo.

\section{Según García Aretio y Medina Rubio} (1993):

Es importante la regulación del proceso de intervención pedagógica en esta modalidad, que se basa en los sistemas de comunicación didáctica entre el docente y los estudiantes, a través del correspondiente diálogo, habitualmente mediado, sea éste simulado, real, síncrono o 
asíncrono. (Pág.56)

Un soporte importante para que esto se haga viable poco a poco ha sido la consideración de las TIC (Tecnologías de Información y Comunicación), las cuales han sido aplicadas de acuerdo con las políticas de cada institución educativa.

René Florido Bacallao y Marilyn Florido Bacallao (1993) comentan que:

La integración de las TIC (materiales multimedia, videoconferencias, TV digital) al sistema de enseñanza, favorece el desarrollo de diversas actividades de postgrado, sin que estén presentes simultáneamente en el mismo lugar todos los factores involucrados en el proceso educativo. De igual forma, queda implícita, la interrelación que debe existir entre estudiantes y profesores en este tipo de enseñanza, donde se deben utilizar medios como videoconferencias, chat, foros de debate, correo electrónico, internet, entre otras. (Pág.3)

La interrelación entre el estudiante y el docente es uno de los aspectos que debe mantenerse fortalecido por las TIC, sumado a las estrategias que plantee el docente para seducir la participación de los estudiantes en un entorno no presencial. De esta forma, se resalta el papel del docente como importante para fomentar una interacción constante durante el periodo de participación en un curso o materia.

Según García (1999) se considera lo siguiente:

Los componentes que interaccionan en un escenario virtual, donde se ha de aplicar la metodología de enseñanza/aprendizaje más adecuada, de acuerdo con:

- El tipo de estudiantes destinatarios de la formación;

- El tipo o modalidad de comunicación didáctica de que se puede disponer dentro de la norma o modelo educativo propuesto;

- El tipo de estructura organizativa en el que se integra su docencia, y

- El tipo de tareas de docentes que expresamente se le requieren dentro de la modalidad educativa diseñada. (Pág. 6)

Podemos diferenciar los siguientes componentes de acuerdo con el trabajo expuesto por García (1999) en la siguiente figura:

\section{Figura $\mathbf{N}^{\circ} 1$ \\ Componentes del sistema de educación a distancia según el modelo de Lorenzo García Aretio}

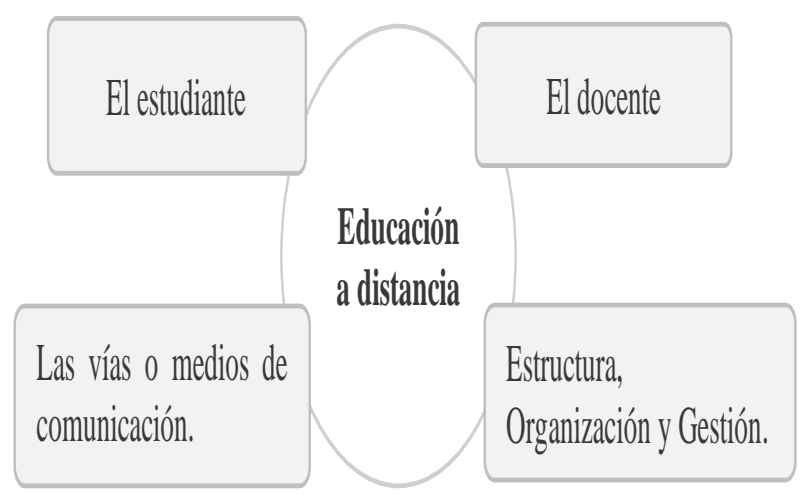

Fuente: Lorenzo García Aretio. Fundamento y Componentes de la Educación a Distancia Elaboración: Propia

Las tecnologías de información implican un soporte importante para mantener la comunicación entre los elementos participantes del proceso de enseñanza aprendizaje, pero la manera de presentarse en cada situación ha dependido de lo que cada organización ha decidido para soportar la educación. Existen sistemas más abiertos, 
los cuales comprenden comunidades más grandes y con una flexibilidad superior en la interacción frente a otros sistemas, que si bien es cierto regulan mejor el tema de evaluación, son más rígidos en la navegación y comprenden una comunidad más limitada, que por lo general obedecen a un programa planificado.

Uno de los aspectos que se pudo observar en algunos de estos sistemas educativos virtuales más cerrados fue el bajo nivel de colaboración, que puede deberse a la poca flexibilidad del entorno, a la desestimación del sistema por parte del docente o la falta de conocimiento para manejar los recursos. Al margen del problema independientes de cada organización para propiciar un ambiente colaborativo, es importante resaltar que estos entornos más cerrados se han visto robustecidos progresivamente en el tiempo por la tecnología ante la dificultad señalada, es decir se han brindado mas recursos para poder trasladar las estrategias del docente a un sistema más cerrado y poder generar una mayor participación.

\section{SISTEMAS TRADICIONALES Y CONTEMPORÁNEOS PARA SOPORTAR LA EDUCACIÓN A DISTANCIA}

Después de haber descrito algunas referencias con relación a sistemas educativos virtuales, la presente investigación toma una figura comparativa planteada en la tesis de investigación "Diseño de una Comunidad Colaborativa en los Sistemas Educativos Virtuales Tradicionales", desarrollada por el autor de este artículo. El trabajo de investigación tomado como referencia clasifica los sistemas educativos virtuales bajo dos enfoques con características propias. Se muestra a continuación aquellas características:
Figura 2:

\section{Comparación de Sistemas Tradicionales y Sistemas Contemporáneos}

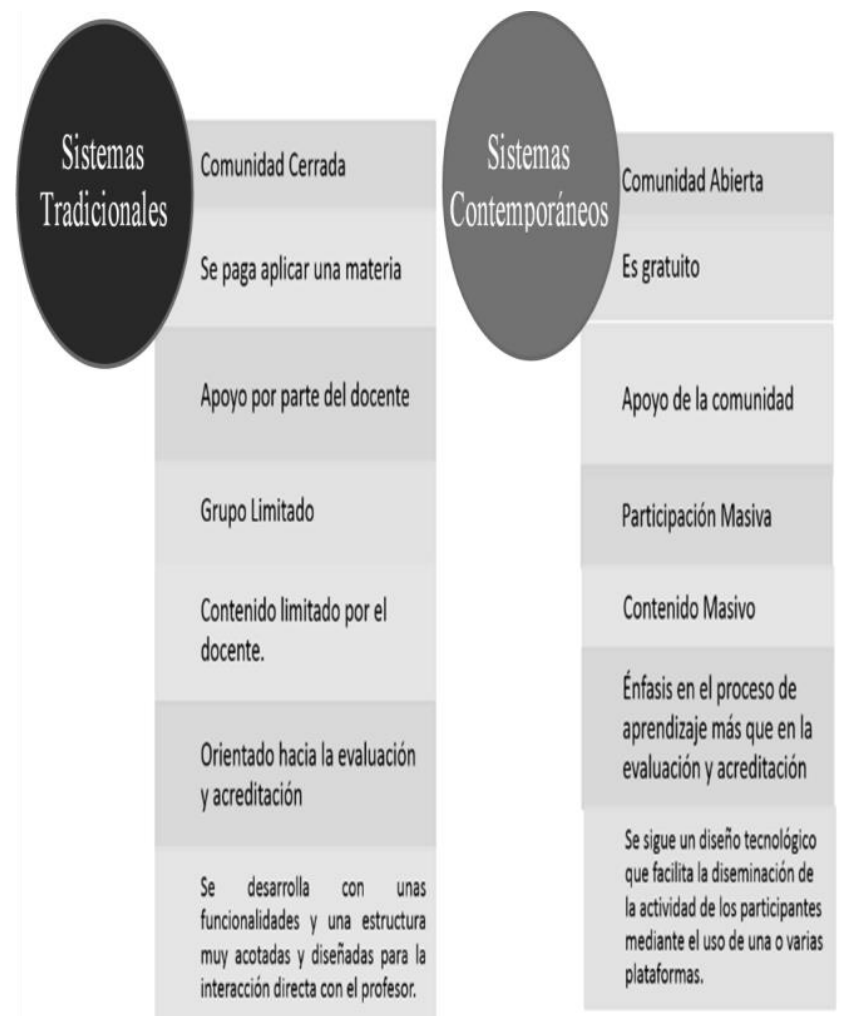

Fuente: Franklin Steve Salazar Visurraga.

Diseño de una Comunidad Colaborativa en los Sistemas Educativos Virtuales Tradicionales.

Elaboración: Propia

El trabajo de investigación de referencia define a los sistemas tradicionales como aquellos sistemas estructurados que tienen una comunidad más limitada para el desarrollo de un aprendizaje virtual o en línea. Por otro lado, define a los sistemas contemporáneos como aquellos sistemas más flexibles que albergan una comunidad más amplia para el desarrollo de un aprendizaje virtual.

Bajo estos dos enfoques, el trabajo de investigación de referencia explora el componente colaboración para determinar la tendencia de una realidad y brindar las respectivas recomendaciones para mejorar el concepto de diseño de los entornos virtuales tradicionales en el Perú.

El factor colaboración resulta importante 
para la construcción de conocimiento y por ende resulta esencial reunir los recursos que permitan al estudiante interactuar constantemente. Las dinámicas y trabajos grupales contribuyen a la consolidación de nuevos conceptos en un entorno presencial, por lo tanto, en un entorno virtual debería conservarse la misma idea para no caer en el único sentido de ser solo un repositorio de información. (Pazos, Pérez \& Salinas, 2001).

Si bien es cierto la colaboración es esencial para el desarrollo de aprendizaje, es un elemento que debe verse fomentado y potenciado por la tecnología si es que se piensa llevar el trabajo de la enseñanza a una plataforma virtual. Algunos escenarios reúnen los recursos para potenciar la colaboración y generación de conocimiento mas no reúne la participación necesaria del moderador para generar las actividades de interacción. En este caso podríamos situarnos en el tipo de sistema tradicional expuesto anteriormente, en la cual la participación de los estudiantes en parte se debe a lo que el docente o moderador proponga en el entorno virtual. El docente o moderador como parte de un entorno virtual debería reunir el conocimiento necesario para usar los recursos de la plataforma, aunque a veces no suele verse así debido a las pocas actividades que se plantean para el estudiante.

Al rescatar el elemento colaboración como fundamental dentro de una comunidad virtual, podemos situarnos en el otro tipo de sistema definido por la tesis de investigación mencionada, debido que sugiere que una estructura más flexible con mayor posibilidad de interactuar para fomentar un mejor aprendizaje. La idea de comunidad no puede estar aislada del factor colaboración, y más aún si se relaciona a un entorno virtual, donde debe siempre procurarse dar las condiciones más óptimas para que el estudiante no solo descargue información útil, sino también pueda compartir experiencias e interactuar con los demás participantes.

En la opinión de Cabero (2006): "Las comunidades virtuales se relacionan directamente con aspectos de sociabilidad e interacción social entre sus miembros, el que requiere un envolvimiento colaborativo y no de aislamiento" (Pág. 5). La participación es vital dentro de una comunidad para fortalecer el aprendizaje, como lo señalaban Dillenbourg, Poirier \& Carles (2006), al rescatar la necesidad de saber y de compartir. Dicha necesidad se convierte en una de las motivaciones principales para ser parte activa de un entorno, el cual debería sostenerse en la colaboración.

Ciertamente las formas colaborativas sostienen el aprendizaje en grupos porque los participantes cooperan entre ellos para generar conocimiento nuevo o fortalecer lo aprendido, lo cual incrementa la motivación para seguir involucrándose en actividades constructivas que implican a su vez habilidades cognitivas, como menciona Mc.Connel (2006).

La rama del derecho implica una constante interacción y participación de los estudiantes por la naturaleza que envuelve dicha especialidad. La enseñanza del derecho se ha caracterizado por ser impartida a través de clases presenciales, donde el docente dirige la clase, siendo pocos los profesores que han adoptado herramientas tecnológicas para utilizarlas en la docencia. A pesar de ello, se concibe a los estudiantes como elementos que participan constantemente para incrementar sus conocimientos mediante actividades de retroalimentación.

Por ello resulta interesante para el presente artículo evaluar el nivel colaboración en un sistema utilizado para impartir enseñanza del derecho. Por lo general las instituciones educativas apuestan por un tipo de sistema 
más estructurado, el cual se encuentra dentro del tipo de sistema tradicional, definido por la tesis de investigación de referencia.

Independientemente del tipo de sistema que una institución educativa elija para desarrollar sus programas, el escenario debe estar diseñado para el estudiante, porque es el elemento que debe verse más beneficiado en todo el modelo educativo.

\section{IMPORTANCIA DEL DISEÑO DE UN ENTORNO VIRTUAL CENTRADO EN EL ESTUDIANTE}

Al hablar de entorno virtual, se rescata el significado de campus virtual, el cual se ve reflejado en el uso de plataformas $e$ learning en la enseñanza universitaria en estos últimos tiempos. En este espacio virtual que puede funcionar como apoyo o como un curso en sí, se llevan a cabo tareas que promueven el aprendizaje. Para ello las tecnologías de información y comunicación (TIC) juegan un papel importante, debido a que siempre con las innovaciones que surgen se busca que el espacio virtual sea el más apropiado para que se genere una enseñanza de calidad y no se extrañe mucho a la enseñanza tradicional en las aulas.

Por otro lado, el campus virtual asocia la idea de universidad virtual como lo expresaba Ramboll (2004), porque hace referencia no solo a espacios relacionados a la enseñanza - aprendizaje, sino a los que se relacionan a la gestión y organización de todas las actividades y procesos que suelen percibirse en un centro educativo superior, independientemente de la rama académica.

Según Ramboll \& Romiszowski (2004), el objetivo institucional es importante para definir el camino del diseño de un campus virtual, el cual está delimitado por recursos y políticas organizacionales.
Fernández-Pampillón (2009) agrupa estos parámetros en tres dimensiones: la institucional, la tecnológica y la didáctica para un mejor entendimiento:

- La dimensión institucional hace referencias a la estructura de la organización, normas de funcionamiento y políticas que engloban el desarrollo del campus virtual.

- La dimensión tecnológica, hace referencia a la parte tecnológica que soporta el desarrollo del campus virtual.

- La dimensión didáctica, se refiere a las metodologías y políticas para el uso del $e$ learning por parte de los usuarios. (Pág. 16)

Estos escenarios, que sostienen la comunicación entre los participantes del proceso de enseñanza - aprendizaje, tienen que estar diseñados para que el estudiante sea el más beneficiado y pueda tener las posibilidades de sentirse acompañado en el proceso de construcción de conocimiento. De igual manera que en los escenarios presenciales, los participantes apuntan a trabajar en un escenario colaborativo como una comunidad, los entornos virtuales no deben perder esa perspectiva desde la concepción de su diseño.

\section{CONSIDERACIÓN DE LA EDUCACIÓN VIRTUAL COMO ALTERNATIVA EN LA ENSEÑANZA DEL DERECHO}

La modalidad de educación virtual o a distancia cada vez más forma parte de las alternativas que las instituciones educativas utilizan para sus programas. Esta modalidad incluye estrategias de enseñanza y aprendizaje; y ha sido entendido en el tiempo de diferentes formas como tele 
formación, telemática educativa, teleeducación, formación multimedia, aprendizaje a distancia, entre otras, como expresa Florido Bacallao (2003).

La modalidad de educación virtual al surgir como alternativa para el aprendizaje fue escalando a distintos niveles. Los programas establecidos por las instituciones fueron incorporando las TIC para soportar sus procesos de enseñanza y brindar un servicio más al alcance del usuario interesado. Podemos reconocer dos tipos de usuarios muy relacionados en el proceso de enseñanza y aprendizaje, por un lado, el docente que debe utilizar las herramientas tecnológicas dentro de su plan de enseñanza y por otro lado el estudiante quien debe participar de las actividades propuestas por el docente soportadas por dichas herramientas. Estas herramientas se hacen más apropiadas cuando adquieren la figura de entornos especializados para desarrollar actividades que permitan fortalecer el aprendizaje.

Entre las distintas manifestaciones de las TIC, los entornos virtuales fueron considerados de gran ayuda al momento de acompañar el trabajo del docente. Aunque estos medios aun suponen cierta resistencia para insertarlo como una alternativa, existen cada vez más instituciones educativas $\mathrm{u}$ organizaciones que apuestan por este medio para soportar sus programas académicos o capacitaciones.

Para Florido Bacallao (2003):

A pesar de la diversidad de soluciones que se suscitan como apoyo a diversas áreas de la educación, ninguna soslaya la integración de las TIC (materiales multimedia, videoconferencias, TV digital) al sistema de enseñanza, para desarrollar diversas actividades, sin que estén presentes simultáneamente en el mismo lugar todos los factores involucrados en el proceso educativo. De igual forma, queda implícita, la interrelación que debe existir entre estudiantes y profesores en este tipo de enseñanza, donde se deben utilizar medios como videoconferencias, chat, foros de debate, correo electrónico, internet, entre otras. (Pág. 3 y 4 )

La tendencia a potenciar la formación semipresencial o completamente virtual mediante las TIC es coherente con las necesidades que se han ido manifestando en el tiempo hacia el mejor escenario del estudiante como menciona De la Torre (2010).

Muchas áreas en la educación se ven favorecidas por el uso de plataformas tecnológicas al momento de iniciar sus programas, aunque pueden diferir en sus formas debido al génesis del diseño. Este diseño debería ser preferentemente orientado al trabajo colaborativo más que un simple repositorio de contenido. Presencialmente se generan muchas actividades de interacción en un ambiente de clases, esto implica que el estudiante pueda construir conocimiento nuevo mediante la participación y orientación del docente.

A pesar de que algunas plataformas tienen ese fin y diseño, solo son utilizadas como repositorios de información debido a la desestimación de la misma como herramienta colaborativa. Esto puede deberse al desconocimiento del funcionamiento de la herramienta en su totalidad, poca incidencia en la capacitación del docente facilitador o simplemente una actitud reacia al cambio de tomar nuevas soluciones de apoyo en la enseñanza.

Una de las áreas que despertó el interés de investigación para el presente artículo fue la enseñanza del derecho, debido a que es un tipo de enseñanza caracterizada por ser impartida a través de clases presenciales mayormente, donde el docente suele tener las iniciativas de las actividades. 
Aunque en un principio fueron pocos los profesores que han adoptado herramientas tecnológicas para utilizarlas en la docencia, hoy en día se pueden apreciar muchos programas del derecho que son soportados por plataformas tecnológicas, lo cual indica que no existe un total divorcio de la especialidad con las tecnologías, puesto que de alguna forma son útiles para soportar contenidos y actividades. Por consiguiente, podrían evaluarse mejoras desde otros aspectos como la colaboración en el escenario, lo cual si resulta importante impulsar para reducir el porcentaje de aquellos que sostienen que los escenarios virtuales no pueden formar parte de las metodologías en la enseñanza.

\section{EL SENTIDO DE COMUNIDAD BASADA EN LA COLABORACIÓN DENTRO DE LA ENSEÑANZA VIRTUAL DE LA RAMA DEL DERECHO}

El sentido de comunidad es un concepto que ha evolucionado durante el tiempo manifestándose en distintas realidades. Como bien afirma Barab (2003) "Existe una extensa historia sobre el concepto de comunidad. Diversos científicos sociales han caracterizado las comunidades de diversas maneras para entender diversos fenómenos sociales, basados en diversas filosofias sociales" (Pág. 197 - 201).

Meirinhos y Osório (2009) sostienen que:

En el ámbito educativo y formativo es cada vez más frecuente la utilización de la palabra "comunidad" asociada a otras palabras o expresiones, algunas ya de utilización frecuente $y$, otras, menos conocidas: comunidades de aprendizaje, comunidad virtual de aprendizaje, comunidad de práctica, comunidades deslocalizadas,

\begin{abstract}
comunidades de interés, comunidades online, comunidades de investigación o indagación, comunidades en red, ciber comunidades, comunidades elearning, etc. El concepto de comunidad puede variar bastante conforme los contextos de utilización. Es un concepto extremamente polisémico, relacionado con las multiplicidades históricas de las realidades sociales y organizacionales (Págs. 45 - 60).
\end{abstract}

Es común llevar el concepto de comunidad en entornos de formación o educación. Las instituciones educativas deben sostener sus programas en un trabajo colaborativo y más aún cuando se trata de enseñanza superior. Cuando el contexto marca una enseñanza superior se tiene en cuenta a los estudiantes y profesores que se ven involucrados en actividades pedagógicas, sea presencial o a distancia. Por lo general el aspecto colaborativo se acentúa más en un escenario presencial pero no debe perderse esa idea cuando el escenario es virtual debido a que debe priorizarse la interacción constante reflejada en el compartir de recursos y colaboración de los participantes, como lo explicaba Daelle \& Brassard (2003).

El concepto de comunidad ha evolucionado en los últimos tiempos debido a las necesidades de cambiar el paradigma tradicional, lo cual conlleva a proponer soluciones innovadoras que atiendan las limitaciones en el campo educativo independientemente de la rama o especialidad (Coll, 2004). En el contexto virtual se han presentado algunas limitaciones o problemas en cuanto a la flexibilidad del escenario. Esto se debe al débil concepto de comunidad colaborativa que tienen desde su concepción o diseño.

La idea de comunidad virtual se ha insertado en las distintas ramas educativas con el tiempo y ha enfrentado desafíos para 
ser el mejor escenario del estudiante. Este escenario se entiende como un entorno organizado y estructurado, el cual es soportado por un sistema de comunicación para albergar la interacción de los participantes, como lo mencionó Daele (2004). Desde esa premisa debería partirse para iniciar las propuestas de diseños de espacios virtuales orientados a la educación debido a que la interacción de los participantes va a depender de la flexibilidad proporcionada en la parte tecnológica y del ambiente de presencialidad y acompañamiento percibido. Dentro de todo ello, el rol del docente tiene la misión de utilizar los recursos tecnológicos para establecer una comunicación con el estudiante adoptando un perfil más innovador en un escenario diferente.

La comunicación en un entorno virtual es esencial para conseguir la interacción entre los miembros participantes y fortalecer la confianza con el uso de nuevos recursos, los cuales deben ser gestionados por el docente al momento de establecer y programar actividades.

Garrison y Anderson (2011) agregan que la comunicación soportada por las tecnologías tiene que desarrollarse de manera abierta, generando respuestas pertinentes y constructivas a las cuestiones planteadas por los demás integrantes de una comunidad que siempre está en busca de conocimiento nuevo cuando se sienten comprometidos.

Gutiérrez-Santiuste y Gallego-Arrufat (2017) sostienen que:

Dentro de un escenario virtual la comunicación abierta está relacionada con la interacción, la cual debe acogerse a las dinámicas grupales para sostener un clima basado en una cercanía social que apoya y promueve el intercambio de ideas dentro de la comunidad, generando aportaciones al conocimiento de todos los participantes. (Pág. 14)

Según la investigación realizada por Elba Gutiérrez-Santiuste y María Jesús GallegoArrufat se observó en una muestra analizada la importancia de la comunicación dentro de un escenario virtual. Esta comunicación se reflejó mediante el uso foros, chats y un sistema de correo, los cuales fueron utilizados como medios soportados por las tecnologías para conservar la interacción entre el alumnado. En este aspecto el docente tuvo un rol importante porque tuvo el papel de guía con los participantes, los cuales se sintieron comprometidos en un entorno diferente. En aquella investigación se rescató el lado afectivo como trascendental para consolidar la interacción porque tuvo una incidencia importante en el grupo de estudiantes debido a que no se sintieron aislado escenario diferente.

El sentimiento de estar en una comunidad basada en la colaboración fue esencial para potenciar el compromiso de trabajar constructivamente para fortalecer el aprendizaje. Como lo mencionan Chiecher y Donolo (2013): "las intervenciones sociales reflejarían la manifestación de mecanismos ligados al establecimiento de condiciones para una interacción estimulante a través de expresiones de refuerzo, ánimo o apoyo entre los miembros" (Pág. 49).

El sentido de comunidad es importante, pero debe estar basado en la colaboración. Conforme a Coll (2004), las comunidades de aprendizaje pueden incrementar considerablemente su eficacia mediante el uso de las tecnologías, debido a que ello potencia el soporte de comunicación e interacción. La evolución de la tecnología ha sido importante en cada rubro de la sociedad y ello no es ajeno a la rama del derecho, específicamente cuando se habla 
de enseñanza.

El dialogo o la discusión forman parte de la construcción del conocimiento en un escenario presencial, pero al contar con recursos de las tecnologías de información y comunicación las instituciones educativas proponen transmitir dicha esencia en programas virtuales para tener un mejor alcance con el objetivo de formar una comunidad de aprendizaje mediante la interacción constante (Guri-Rosenblit y Gros ,2011). Osorio y Duart (2011, Pág.71) definen que "La interacción puede potenciar la construcción individual y colaborativa de conocimiento siempre que se cumplan ciertas condiciones y características en los diseños y el desarrollo de las actividades tanto con herramientas síncronas o asíncronas de comunicación".

Por otro lado, debe entenderse que, si bien las comunidades físicas tienen una manera de relacionarse, las comunidades virtuales tienen otra lógica, pero de igual forma no deben perder la sociabilidad mediante mecanismos de organización como lo sostiene Salinas (2003).

Merinhos \& Osório (2009) sostienen que:

Aprender colaborativamente, en ambientes virtuales, requiere algo más que competencias de aprendizaje individual. Uno es responsable por el aprendizaje de los otros también, con los cuales, de alguna manera se relaciona. El aprendizaje colaborativo en ambientes virtuales establece una nueva relación entre los participantes del proceso de aprendizaje, una vez que el aprendizaje colaborativo es más exigente que el aprendizaje individual. En el ambiente virtual, la colaboración, requiere una mediación tecnológica y el alargamiento en el espacio y en el tiempo del relacionamiento $\mathrm{y}$, por otro lado, requiere un proceso interactivo intenso y regular de envolvimiento cognitivo (Pág. 45 60).

La colaboración tiene su base en la colaboración, pero necesita que se desarrolle en un nivel determinante para sostener una comunidad de aprendizaje constante para propiciar una creciente participación en el entorno virtual. (Zhao, Sullivan y Mellenius, 2013).

Teniendo en cuenta lo anteriormente expuesto podemos decir que la rama del derecho necesita que se establezca una comunicación abierta teniendo como soporte a las tecnologías de información y comunicación. La presencia social siempre fue importante en la rama del derecho para poder afianzar los conocimientos de campo $\mathrm{y}$ fortalecer el aprendizaje mediante el intercambio de experiencias.

Es importante que el estudiante perciba un acompañamiento del guía y de los demás participantes dentro del proceso de aprendizaje en un entorno virtual. La presencia percibida conduce la confianza de los participantes hacía una interacción productiva consiguiendo de esta manera la figura de una comunidad de aprendizaje. La presencia social como elemento motivador ha ido fortaleciéndose durante las diversas presentaciones de entornos virtuales (Garrison Anderson y Archer, 2010). La interacción progresiva disminuye el sentimiento de soledad en un ambiente diseñado para la colaboración, aunque también es necesario que el docente promueva la participación constante como comunidad (Mc. Innerney y Roberts, 2004).

La rama del derecho necesita conservar ese sentido de comunidad basado en la colaboración para el aprendizaje. Por naturaleza es un campo con interacción constante y más allá que pueda ser 
soportado por un sistema virtual que registre puntuaciones a las actividades propuestas, requiere preservar ese acompañamiento para el debate constante e intercambio de ideas. Una comunidad que se mantiene facilita y contribuye al aprendizaje porque garantiza un grupo cohesionado, en cual se destaca la confianza entre los participantes para la construcción de conocimiento. La percepción de estar en una comunidad brinda un salto de calidad a un entorno que en un principio encontró adversidades en relación a su aceptación como parte de la enseñanza. (Remesal y Colomina, 2013).

Para potenciar la enseñanza en la rama del derecho se necesita que los estudiantes también estén comprometidos, pero ello requiere que se genere un fuerte sentido de comunidad para motivar la participación en la acción formativa (Valverde, 2013). La dedicación del estudiante dentro de un entorno virtual es muy importante, es decir, el hecho de que pueda comprometerse para trabajar con los demás participantes mediante el uso de los recursos de un sistema virtual es de suma importancia para reflejar una organización de trabajo. En este aspecto la capacidad de autonomía resalta mucho, debido a que los usuarios traen sus propias experiencias para contribuir con la formación. (Henri \& Basque, 2003)

$\mathrm{Si}$ bien es cierto pueden existir comunidades más cerradas que otras, lo más importante es que cuente con los recursos que permitan la interacción constante del estudiante, más allá del tipo de escenario en el cual este desarrollándose aprendizaje.

\section{METODOLOGÍA \\ INVESTIGACIÓN}

DE

Teniendo en cuenta que el artículo rescata la importancia del factor colaboración para desarrollar un aprendizaje optimo en la rama del derecho, se establecen acciones para determinar una tendencia. Se toma como referencia un análisis realizado por la tesis de investigación mencionada anteriormente, en el cual se concluye que los sistemas educativos virtuales tradicionales, cuyos escenarios son más rígidos y estructurados, conservan un nivel de colaboración menor con respecto a otros sistemas educativos virtuales que tienen comunidades más grandes y flexibles.

La investigación realizó un análisis cuantitativo para medir la media de las puntuaciones y la media con el fin de ver dispersión en los resultados. También realizó un análisis cualitativo para describir las puntuaciones obtenidas. Estas puntuaciones fueron categorizadas en satisfecho e insatisfecho, con lo cual se utilizó la proporción como medida para interpretar los resultados.

Se elige una población con características similares a la población tomada en la tesis mencionada para evaluar que tanto ha variado el nivel colaborativo en un sistema educativo virtual tradicional.

\section{Determinación de población y muestra}

La población elegida para determinar el valor de comunidad basada en la colaboración en la presente investigación fue el curso de Temas de Teoría del Delito que se dictó en la Facultad de Derecho de la Universidad de San Martin de Porres, desde el 18 de marzo hasta el 11 de mayo del 2019.

Los participantes mayormente fueron miembros de la Policía Nacional del Perú y se asume una consciente responsabilidad de su parte para enrolarse con las actividades propuestas por el docente dentro la plataforma. El número de participantes matriculados en el diplomado fue de 32 , el cual es considerado como población.

El tamaño de la muestra fue de 22 estudiantes para analizar el valor 
colaborativo de comunidad. El factor colaboración resulta vital dentro de una comunidad virtual orientada al aprendizaje, y más aún si esta comunidad pertenece a la rama del derecho.

El tipo de investigación es de naturaleza descriptiva debido a que se selecciona $\mathrm{y}$ describe una serie de aspectos relacionados con la dimensión colaboración, medido a través de foros de discusión, comunicación fluida e interacción entre los compañeros, y mediremos cada una de ellas independientemente. Los resultados nos servirán para comparar ambos sistemas educativos virtuales en su aspecto comunidad basada en la colaboración.

El diseño de investigación fue transversal porque se realizó en un momento del tiempo, teniendo como instrumentos de recolección de datos el cuestionario y como técnica a la encuesta.

El procesamiento y análisis de datos se realizó mediante el software estadístico SPSS $^{1}$, donde se realizó las clasificaciones de las variables de análisis, para después realizar las tabulaciones.

Los escenarios representativos para poder establecer un análisis en con respecto a la dimensión colaboración:

- La Universidad de San Martin de Porres - Lima, Perú

$\checkmark$ Facultad de Derecho - Plataforma Moodle

- Diplomados Virtual Presencial 2014 (Agosto Noviembre)

- La Universidad de San Martin de Porres - Lima, Perú

$\checkmark$ Facultad de Derecho - Plataforma

\footnotetext{
${ }^{1}$ Statistical Package for the Social Sciences. Es un programa utilizado para realizar la captura y análisis de datos para crear tablas y gráficas con data compleja.
}

\author{
Moodle \\ - Curso Virtual - Presencial \\ 2019 (Marzo Mayo)
}

\section{Prueba de Hipótesis}

Se toma en cuenta la prueba de ChiCuadrado para probar el objetivo de la investigación. Se puede entender de esta prueba como un esquema en el cual comparas lo esperado con lo obtenido en base a valores que se obtienen. Suponiendo que la hipótesis nula sea cierta se procede a calcular la frecuencia que se espera en cada intervalo de valores.

Se tiene en cuenta el tamaño de la muestra y la probabilidad del intervalo de valores de la hipótesis nula para hacer la prueba o validez.

\section{Estadística de prueba relacional}

Según el trabajo de Ramos \& Ticona (2016) se puede desprender la siguiente formula del estadístico de prueba (Pág. 81):

El estadístico de prueba se basa en las diferencias entre la Oi y Ei. Esto se define como:

$$
X^{2}=\sum_{i=1}^{n} \frac{\left(O_{i}-E_{i}\right)^{2}}{E_{i}}
$$

Donde,

$\mathbf{X}^{\mathbf{2}}=$ Estadístico Chi Cuadrado

$\mathbf{O i}=$ Frecuencia Observada

$\mathbf{E i}=$ Frecuencia Esperada

$\sum=$ Sumatoria

Este estadístico tiene una distribución Chi-Cuadrado con n-1 grados de libertad si $n$ es suficientemente grande, es decir, si

Tiene la capacidad de gestionar grandes volúmenes de datos y es capaz de llevar a cabo análisis de texto. 
todas las frecuencias esperadas son mayores que 5. En la práctica se tolera un máximo del $20 \%$ de frecuencias inferiores a 5 .

$\mathrm{Si}$ existe concordancia perfecta entre las frecuencias observadas y las esperadas el estadístico tomará un valor igual a 0 ; por el contrario, si existe una gran discrepancia entre estas frecuencias el estadístico tomará un valor grande $\mathrm{y}$, en consecuencia, se rechazará la hipótesis nula. Así pues, la región crítica estará situada en el extremo superior de la distribución ChiCuadrado con k-1 grados de libertad. (Fabela, 2015, Pág.5)

\section{ANÁLISIS DE LOS RESULTADOS Y PRUEBA DE HIPÓTESIS}

Realizaremos un primer análisis descriptivo de los ítems (preguntas) de la muestra actual, para después medir la relación que existe de cada ítem con la dimensión comunidad y finalmente realizaremos la prueba de Chi-Cuadrado para probar la hipótesis que el porcentaje de satisfacción con la Dimensión Comunidad no es diferente en ambas muestras.

\section{Tabla $\mathrm{N}^{\circ}$ 1:Estadísticas Descriptivas de la Dimensión Comunidad}

\begin{tabular}{lcccc} 
Dimensión Comunidad & Media & Mediana & Mín. & Máx. \\
\hline $\begin{array}{l}\text { ¿Considera se establecen for os de } \\
\text { discusión en la plataforma virtual } \\
\text { durante el curso para su debate? }\end{array}$ & 3.90 & 4.00 & 1.00 & 5.00 \\
\hline $\begin{array}{l}\text { ¿Considera se establece una } \\
\text { comunicación fluida entre el } \\
\text { docente/moderador y estudiantes } \\
\text { mediante la plataforma virtual? }\end{array}$ & 3.87 & 4.00 & 1.00 & 5.00 \\
\hline \\
$\begin{array}{l}\text { ¿Considera que sus compañeros } \\
\text { en la plataforma virtual colaboran } \\
\text { con usted para absolver sus dudas } \\
\text { y consolidar sus conocimientos? }\end{array}$ & 3.81 & 4.00 & 1.00 & \\
\hline
\end{tabular}

Fuente y Elaboración: Propia
En la Tabla $\mathrm{N}^{\circ} 1$ se observa las medidas descriptivas como resultado, la puntuación media en cada pregunta no dista mucho de su mediana con lo que la percepción es casi homogénea, sin embargo, hay valores extremos en cada una de las preguntas.

Figura $\mathrm{N}^{\circ} 3$

\section{Relación entre Foro de Discusión y la Dimensión Comunidad Colaborativa}

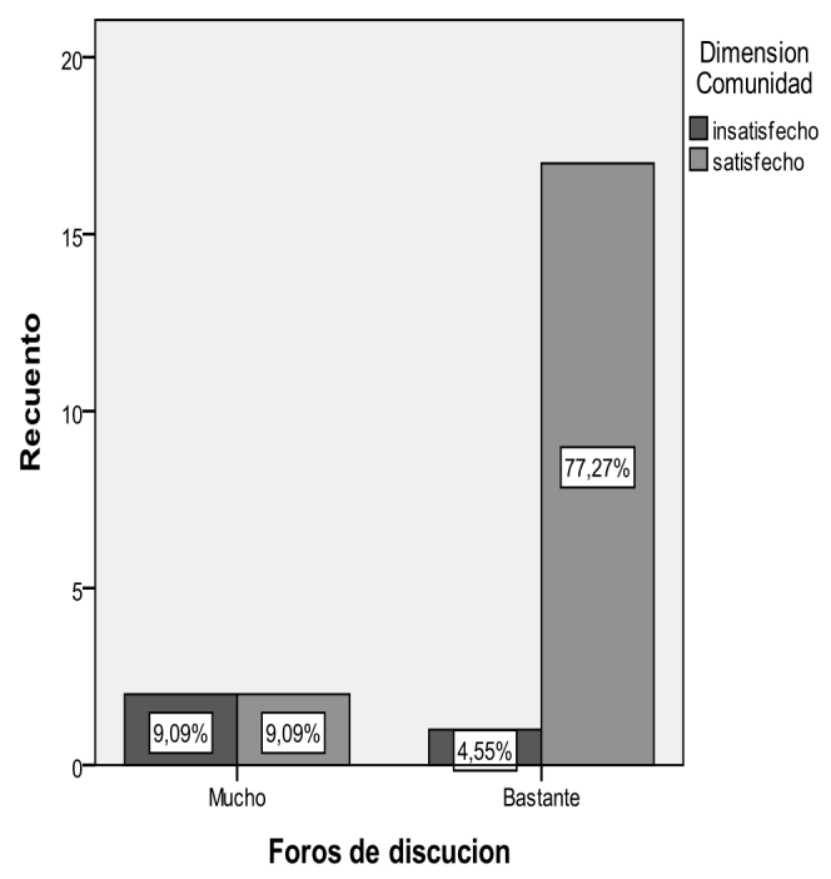

Fuente y Elaboración: Propia

En la Figura $\mathrm{N}^{\circ} 3$ muestra como en la categoría mucho de foros de discusión, la satisfacción respecto a la dimensión comunidad colaborativa es la misma, sin embargo, cuando está en categoría bastante la participación en los foros de discusión refleja un $77 \%$ de estudiantes satisfechos con la dimensión comunidad colaborativa. 
Figura $\mathrm{N}^{\circ} \mathbf{4}$

Relación entre Comunicación Fluida y la Dimensión Comunidad Colaborativa

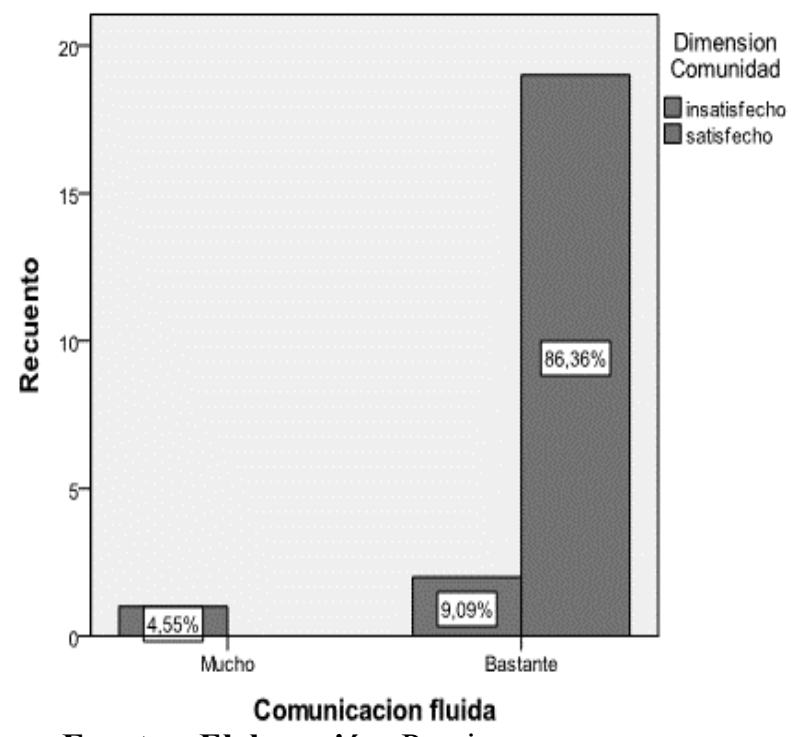

Fuente y Elaboración: Propia

En la Figura $\mathrm{N}^{\circ} 4$ se muestra como en la categoría mucho de comunicación fluida no se aprecia satisfacción por la dimensión comunidad colaborativa, sin embargo, cuando la comunicación fluida está en categoría bastante, el $86 \%$ de los estudiantes están satisfechos con la dimensión comunidad colaborativa.

Figura $\mathbf{N}^{\circ} 5$

Relación entre Colaboración de Compañeros y la Dimensión Comunidad Colaborativa

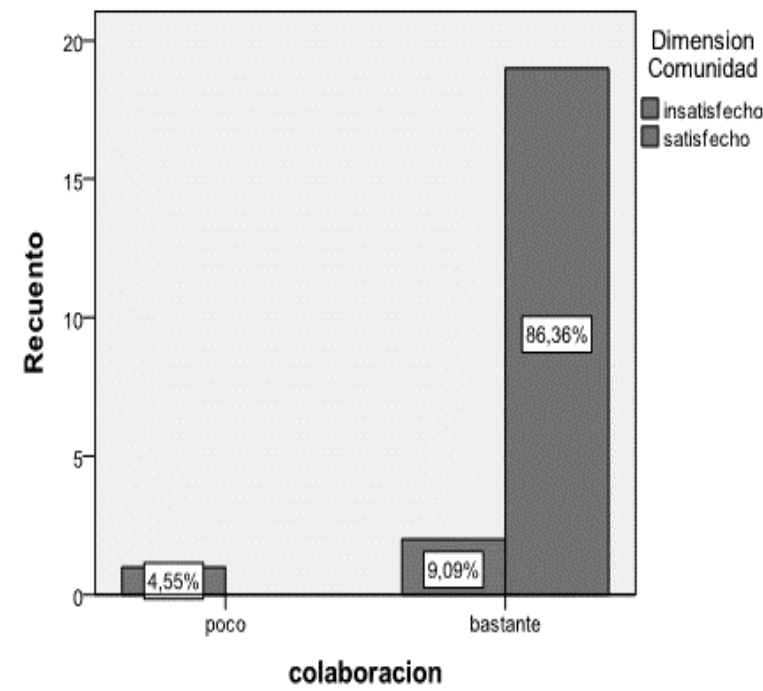

Fuente y Elaboración: Propia
En la Figura $\mathrm{N}^{\circ} 5$ se muestra como en la categoría poco de colaboración de compañeros se aprecia un nivel bajo de satisfacción por la dimensión comunidad colaborativa, sin embargo, cuando la colaboración de compañeros está en categoría bastante, se refleja un $86 \%$ de satisfacción de los estudiantes con la dimensión comunidad colaborativa.

\section{Dimensión Comunidad}

La muestra de 22 estudiantes del Curso de Temas de Teoría del Delito soportado por un sistema virtual tradicional es evaluada respecto a la dimensión comunidad basada en la colaboración. Esta evaluación muestra que el $86 \%$ de los estudiantes están satisfechos con dicha dimensión.

\section{Figura $\mathrm{N}^{\circ} 6$ \\ Dimensión Comunidad basada en la colaboración}

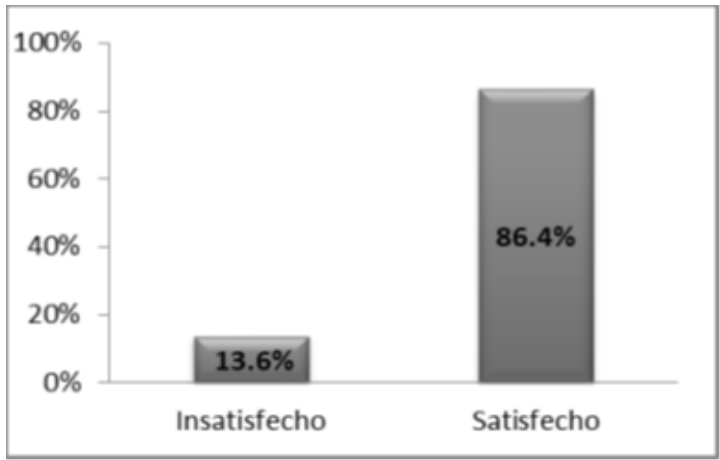

Fuente y Elaboración: Propia

Este resultado es bastante diferente a lo esperado dado que en los sistemas de educación virtual con una composición más estructurada o rígida la satisfacción respecto a la dimensión comunidad suele ser baja, porque no se establece mucha participación en ellos, no hay una comunicación fluida entre todos los participantes incluyendo al moderador, y por último no hay apoyo en resolver dudas por parte de los compañeros. 
Para probar estadísticamente si existe diferencia significativa entre los porcentajes de satisfacción obtenidos en la tesis de referencia respecto a la dimensión comunidad basada en la colaboración y del grupo de 22 estudiantes, realizaremos la prueba de Chi-Cuadrado para establecer o representar el cambio de una cultura de comunidad basada en la colaboración de los sistemas educativos virtuales tradicionales. En ambos casos se representan sistemas que soportan el aprendizaje en la rama del derecho en grupos con mayor experiencia en la materia y con un sentido de responsabilidad, puesto que son estudiantes de ciclos superiores, egresados o profesionales.

\section{Diferencia de Proporciones}

$$
\begin{aligned}
& H_{0}: \pi_{1}=\pi_{2} \\
& H_{1}: \pi_{1} \neq \pi_{2}
\end{aligned}
$$

- Ho: No existe diferencia entre el porcentaje de satisfacción respecto a la dimensión comunidad basada en la colaboración de los estudiantes de la tesis de referencia y del grupo evaluación en este artículo.

- H1: Si existe diferencia (Por lo tanto, se ha desarrollado la cultura de comunidad basada en la colaboración en los sistemas educativos virtuales tradicionales)

\section{Tabla N ${ }^{\circ}$ : Grupo de Muestras y Dimensión Comunidad}

\begin{tabular}{|c|cc|c|}
\hline \multirow{2}{*}{ Grupos } & \multicolumn{2}{|c|}{$\begin{array}{c}\text { Dimensión Comunidad } \\
\text { basada en la Colaboración }\end{array}$} & \multirow{2}{*}{ Total } \\
\cline { 2 - 3 } & Insatisfecho & Satisfecho & \\
\hline Muestra Tesis & 48 & 10 & 58 \\
Muestra Actual & 3 & 19 & 22 \\
\hline Total & 51 & 29 & 80 \\
\hline
\end{tabular}

Fuente y Elaboración: Propia
El cuadro muestra un cambio significativo en los porcentajes de satisfacción respecto a la dimensión comunidad basada en la colaboración. La satisfacción obtenida fue de un $17 \%$ en la tesis de referencia y un $86 \%$ en el grupo de estudiantes del Diplomado de Temas de Teoría del Delito.

\section{Tabla N $N^{\circ}$ : Resultado de la Prueba de

\begin{tabular}{|c|c|c|c|c|c|}
\hline & Valor & $g \mid$ & $\begin{array}{l}\text { Sig. asintótica } \\
\text { (bilateral) }\end{array}$ & $\begin{array}{l}\text { Sig. exacta } \\
\text { (bilaterall) }\end{array}$ & $\begin{array}{l}\text { Sig. exacta } \\
\text { (unilateral) }\end{array}$ \\
\hline Chi-cuadrado de Pearson & $32,977^{\mathrm{a}}$ & 1 &, 000 & & \\
\hline $\begin{array}{l}\text { Corrección por } \\
\text { continuidadb }\end{array}$ & 30,054 & 1 &, 000 & & \\
\hline Razón de verosimilitudes & 33,925 & 1 &, 000 & & \\
\hline $\begin{array}{l}\text { Estadistico exacto de } \\
\text { Fisher }\end{array}$ & & & &, 000 &, 000 \\
\hline $\begin{array}{l}\text { Asociación lineal por } \\
\text { lineal }\end{array}$ & 32,565 & 1 &, 000 & & \\
\hline N de casos válidos & 80 & & & & \\
\hline
\end{tabular} Chi-Cuadrado}

Fuente y Elaboración: Generado mediante el Software SPSS (Statistical Package for the Social Sciences)

Para evaluar el nivel de significación de la prueba, compararemos si el p-value es menor a 0.05 ( $\mathrm{p}$-value < 0.05) lo que indica que la hipótesis nula será rechazada.

Para nuestro caso, el nivel de significación de la prueba Chi-Cuadrado resulta menor que 0.05 lo que indica que rechazamos la hipótesis nula (ho $=$ "No existe diferencia entre la satisfacción respecto a la dimensión comunidad basada en la colaboración de los estudiantes de la tesis de referencia y del grupo evaluación en este artículo").

Por lo tanto, del análisis se desprende que el grupo de estudiantes evaluados han desarrollado la dimensión de comunidad basada en la colaboración dentro de un sistema educativo virtual tradicional (más estructurado y rígido), con lo que los 
estudiantes interactúan más entre ellos y con el profesor o moderador, generando participación en foros de discusión que permite enriquecer el aprendizaje en la rama del derecho.

\section{DISCUSIÓN}

La presente investigación pretende determinar cómo ha evolucionado el concepto de comunidad basado en la colaboración en los sistemas educativos virtuales tradicionales en la rama del derecho. Para ello, como se mencionó anteriormente, se tomó como referencia la tesis de investigación "Diseño de una comunidad colaborativa en los sistemas educativos virtuales tradicionales", la cual expone la importancia del factor colaboración en una comunidad virtual (plataforma) orientada al aprendizaje. Dicha investigación tomó una comunidad de la rama del derecho, en la cual el aspecto colaborativo no tuvo mucha incidencia con respecto a otros sistemas más flexibles, los cuales fueron soportados por escenarios virtuales más centrados en el estudiante.

Por tal motivo, la presente investigación intenta explorar el aspecto colaborativo en una comunidad virtual con las mismas características de la comunidad estudiada en la tesis de referencia, para poder determinar cómo ha evolucionado el factor colaboración en los sistemas educativos virtuales tradicionales. Debemos recordar que se recoge el concepto de sistemas educativos virtuales tradicionales de la tesis de investigación tomada como referencia, la cual expone a aquellos sistemas como plataformas más estructuradas y con una comunidad más cerrada.

Cabe rescatar que la idea de construcción del conocimiento a través de los propios participantes tiene antecedentes cuando David T. Boven en su trabajo menciona el método Bell - Lancaster, el cual se basa en el principio de que los estudiantes podrían ser maestros de sus compañeros. Una comunidad basada en la colaboración siempre fue considerada importante y se cree necesario conservar esa premisa para el diseño de escenarios virtuales más idóneos para el estudiante, mediante el cual pueda tener una interacción más flexible. También es correcto señalar que el trabajo colaborativo en un escenario virtual depende también del docente o moderador $\mathrm{y}$ de las actividades que proponga, pero en este caso se intenta conocer el factor colaboración desde la percepción del usuario estudiante, mediante una encuesta de tipo Likert debido a que desde el presente artículo también se considera importante al estudiante como fuente para evaluar el factor colaboración del entorno que lo envuelve y más aún si dicho escenario está orientado con la rama del derecho, la cual por tradición involucra mucha participación e interacción. Por tal motivo se toma la rama del derecho como entorno para definir que tanto apoyo tiene desde la tecnología, en este caso desde las plataformas virtuales en el aspecto colaboración.

Es preciso mencionar que el derecho y tecnología no siempre han ido de la mano y en el tiempo distintas experiencias han podido transmitir cierta resistencia del campo del derecho hacia la tecnología. La revista Educación y Cultura cita una reflexión de Carbonell que en su estudio realizó.

Los abogados son, en general, muy reacios al avance tecnológico. No es algo reciente, se ha dado siempre. Los abogados casi siempre llegan tarde a los desarrollos de otras ramas del conocimiento. Esa reticencia o falta de espíritu abierto a la innovación es algo que afecta profundamente al derecho, para cuya actualización a veces hay que esperar demasiados años. El mundo cambia y los abogados parecen resistirse a verlo, o tomar medidas 
para seguirle el paso a la fluctuante realidad. (Carbonell, 2016, Párrafo 3)

Dicha reflexión invita a pensar en la resistencia que puede generarse desde la enseñanza del derecho teniendo en cuenta que vivimos en una sociedad de la información que se caracteriza por los cambios sustanciales que se dan en nuestro entorno y entre ellos la cultura. Por ello, que la invitación intenta evaluar en la rama del derecho que tan positivo resulta el apoyo de las TIC, específicamente las plataformas virtuales que por teoría deberían prevalecer la premisa de brindar el mejor escenario al estudiante. La presente investigación considera que el factor colaboración es esencial para ese escenario y pretende que tanto evolucionó o cambio dicho factor con respecto a la tesis de investigación que se toma como referencia.

Para el desarrollo de la prueba se toma como muestra a un grupo de 22 participantes del curso virtual Temas de Teoría del Delito que se dictó en la Facultad de Derecho de la Universidad de San Martin de Porres, desde el 18 de marzo hasta el 11 de mayo del 2019. Este grupo desarrolló el curso en una plataforma virtual, cuyo escenario corresponde al mismo tipo que la tesis de investigación de referencia analizó. Podemos decir que, por su naturaleza y características, el escenario virtual tomado por el artículo para evaluar el aspecto colaborativo corresponde al tipo de sistemas educativos tradicionales.

La tesis de investigación tomada como referencia clasificó dos tipos de sistemas educativos virtuales: tradicionales $\mathrm{y}$ contemporáneos. Por lo general, las instituciones educativas asumen un tipo de sistema educativo virtual tradicional para el desarrollo de sus programas con una comunidad más limitada, lo cual no implica que no pueda desarrollarse las actividades necesarias para generar retroalimentación y construcción de conocimiento. La poca incidencia de colaboración podría deberse a muchos factores por analizar, pero en principio el artículo pretendió observar si el aspecto colaboración en la muestra seleccionada corrobora la tendencia expuesta en la tesis de investigación tomada como referencia.

Como se dijo anteriormente, el escenario sobre el cual se desempeña la muestra seleccionada para el análisis de este artículo conserva las mismas características de la muestra tomada por la tesis de investigación que funciona como referencia para evaluar la tendencia en el aspecto de colaboración. Se aplicó la encuesta tipo Likert para evaluar el factor colaboración dentro de una comunidad virtual y una vez que se obtuvieron los resultados se comparó con los resultados obtenidos en la tesis de investigación para determinar qué tanta variación hubo en el factor colaboración.

Se utilizó la prueba de Chi- Cuadrado como método estadístico para la prueba relacional, es decir, para determinar si se aprueba o rechaza la hipótesis nula $(\mathrm{ho}=$ "No existe diferencia entre la satisfacción respecto a la dimensión comunidad basada en la colaboración de los estudiantes de la tesis de referencia y del grupo evaluación en este artículo"). El resultado de la prueba estadística relacional muestra un cambio significativo en los porcentajes de satisfacción respecto a la dimensión comunidad basada en la colaboración. La satisfacción obtenida fue de un $17 \%$ en la tesis de referencia y un $86 \%$ en el grupo de estudiantes del curso de Temas de Teoría del Delito. Definitivamente se observa un cambio significativo.

Se había mencionado en los puntos previos que mucho tiene que ver las políticas que las instituciones educativas determinan a la hora de implementar un programa educativo sobre un escenario virtual y al ver que bajo un mismo sistema hubo una 
varianza significativa se recogió las recomendaciones de la tesis de investigación al momento de concluir que el factor colaboración en su muestra era de poca incidencia. Se destacan dos recomendaciones de la tesis de investigación de referencia:

- Concientizar la importancia del sentido de comunidad basado en la colaboración y considerarla en las metodologías que se implementen en los entornos virtuales educativos en adelante. Es decir, cada cierto tiempo aplicaciones muestran nuevas funcionalidades para que sus usuarios estén más en contacto con otros usuarios de su entorno. Ello muestra la realidad de una necesidad de siempre estar en constante relación o interacción. Este caso no es ajeno a los entornos virtuales con fines académicos. El interactuar bajo una buena flexibilidad seria recomendado para un mejor aprendizaje colaborativo.

- Las instituciones educativas que utilizan sistemas educativos virtuales deben evaluar la posibilidad de reestructurar el escenario que presentan para tener el ambiente más apropiado para los usuarios de tipo estudiante, en el cual se priorice el trabajo de grupo, debido a que no debe de perderse la perspectiva de que el estudiante es el más importante en el proceso de aprendizaje.

En base a ellas el presente artículo analiza que tanto cambió en su disposición el entorno que encierra la muestra analizada dentro de la rama del derecho.

Con respecto a la primera recomendación que la tesis de investigación de referencia brindó se puede decir que el curso de Temas de Teoría del Delito contó con una herramienta desde su plataforma virtual que permitió una mayor participación e interacción al momento de establecer sesiones de chat con el tutor o moderador. Por lo tanto, se puede deducir que la innovación y flexibilidad formó parte de la mejora en este caso. Se debe tener en cuenta que tanto el programa educativo analizado por la tesis de investigación de referencia y el programa analizado por esta investigación forman parte de la misma institución educativa en la rama del derecho. Debido a ello se considera que bajo las mismas condiciones se pudieron ejercer mejoras y por ende en la muestra analizada por el artículo se percibió una satisfacción mayor por parte del usuario estudiante.

Con respecto a la segunda recomendación, se puede decir que la encuesta que se aplicó midió otros factores complementarios que dan a entender que hubo un mejor acercamiento del usuario estudiante a la plataforma virtual. El otro componente del escenario, que es el tutor o moderador, tuvo una llegada más acentuada a la hora de intervenir. Técnicamente la conexión no fue tan difícil y hubo una participación en su mayoría, lo cual puede indicar por otro lado que no es tanto el rechazo que puede existir entre la rama del derecho y la tecnología si es que se intenta brindar un mejor acompañamiento al estudiante y si el escenario cuenta con los recursos que permitan una mejor interacción. Entre la participación del moderador y un mejor escenario puede producir un mejor espacio para el desarrollo del conocimiento del estudiante en la rama del derecho, sin descuidar que el escenario debe permitir que el estudiante de la rama del derecho interactúe y pueda relacionarse mejor con los otros compañeros. Quizás este último punto aun es una deuda pendiente para mejorar, pero se observa una mejor aceptación en el grupo analizado. 
El factor colaboración en la muestra analizada por el artículo no tuvo mucha incidencia en ese aspecto porque el nivel de acompañamiento fue bajo según los resultados. En este caso se confirma la importancia de cuidar de que el estudiante como usuario no se sienta aislado en un entorno virtual debido a que por naturaleza necesita de los otros miembros para construir conocimiento, así como también de la debida participación del docente o moderador para guiar la participación mediante las actividades propuestas.

Finalmente, se cree conveniente conservar el elemento colaboración al momento de diseñar o elegir un escenario virtual en la rama del derecho y en todas las demas orientadas a la formación.

Ana Fernández-Pampillón Cesteros en su trabajo de investigación sostiene que el diseño de un escenario virtual debe evaluarse desde tres dimensiones: institucional, tecnológica y didáctica. Es cierto que cada institución educativa puede priorizar una perspectiva más que otra, pero es necesario para mantener un aprendizaje óptimo el factor colaboración entre ellos para brindar un mejor escenario para el estudiante y más aún si nos referimos en la rama del derecho. Desde lo institucional, tecnológico y didáctico debe sostenerse el trabajo en equipo, la flexibilidad y el apoyo esencial de los miembros participantes.

\section{CONCLUSIONES}

Los resultados del análisis mostraron un nivel colaborativo mayor en la muestra seleccionada con respecto a la muestra de la tesis de investigación tomada como referencia para la comparación. El nivel colaborativo se obtuvo mediante la percepción de los mismos participantes. Teniendo en cuenta que ambas muestras se ubicaron dentro del conjunto de sistemas educativos virtuales tradicionales, tuvieron una gran diferencia en su factor colaboración. Si bien es cierto, la tesis de referencia concluyó que este tipo de sistemas educativos virtuales tenían problemas para generar un aprendizaje colaborativo, en este caso el análisis de la muestra seleccionada mostró un resultado alentador con respecto al factor colaboración, el cual es importante en el proceso enseñanza - aprendizaje de la rama del derecho.

Se percibió una mayor participación en el escenario virtual analizado por el artículo, lo cual generó un mejor fortalecimiento del conocimiento, debido a que se tenía el apoyo de los compañeros. Si bien es cierto, en la tesis de referencia se sostuvo que el desarrollo de una comunidad virtual basada en la colaboración se da con mayor satisfacción en sistemas más flexibles y abiertos debido a la gran interacción que sucede, en este caso, que corresponde el análisis a una muestra del sistema educativo virtual tradicional, se observó mejoras en el aspecto colaborativo. Esto puede desprenderse a mejoras que fueron desarrollándose en las propuestas de diseño e incorporación de recursos en esos sistemas educativos virtuales tradicionales en la enseñanza del derecho.

Se vieron iniciativas de mejoras en un tipo de sistema virtual tradicional para la rama del derecho, en el cual se percibió un mejor entendimiento del usuario estudiante para conectarse a las sesiones de chat. Las sesiones de chat son el impulso colaborativo para que los usuarios participantes no pierdan el sentido de comunidad. A su vez, se suma el acompañamiento del docente moderador en la absolución de consultas en el tiempo oportuno. Estos elementos permiten que los estudiantes puedan guiar mejor el nuevo conocimiento teniendo como base la interacción y retroalimentación.

Las dinámicas de trabajo o actividades en un escenario virtual educativo son muy 
importantes para mantener conectados a los participantes y más aún si se ello va acompañado de una flexibilidad proporcionada por la tecnología y aceptación por la organización. Aun se sugieren mejoras desde el diseño de dichos escenarios, pero es importante los avances logrados para fortalecer la consideración de estor recursos tecnológicos como parte de la metodología en la enseñanza del derecho.

\section{FUENTES DE INFORMACIÓN}

\section{Fuentes bibliográficas}

Bretz, R. (1983). Media for interactive communication. London: Sage.

Daelle, A.; Brassard, C. (2003). Communautés d'apprentissage dans l'enseignement supérieur: objectifs et conditions de développement. Congreso Les communautés virtuelles d'apprentissage. 2e colloque de Guéret.

Daelle, A. (2004). Développement professionnel des enseignants dans un contexte de participation à une communauté virtuelle: une étude exploratoire.

Fernández- Pampillón Cesteros, A. (2009). Las plataformas e-learning para la enseñanza y el aprendizaje universitario en Internet. Madrid, España: Universidad Complutense de Madrid.

García Aretio, L.(1989). La educación. Teorías y conceptos - Madrid.

García Aretio, L.; Medina Rubio, R. (1993). Guía didáctica de Teoría de la educación. Madrid: UNED.

Guérin, F. (2004). Le concept de communauté: une illustration exemplaire de la production des concepts en sciences sociales? Paper presented at the $13^{\mathrm{a}}$ Conférence de l'AMI, Normandia . Vallé de Seine.
Henri, F; Basque, J. (2003). Conception d'activités d'apprentissage collaboratif en monde virtuel, en Collaborer pour apprendre et faire apprendre. DEAUDELIN C.

McConnel, D. (2006). E-learning Groups and Communities. New York. McGrawHill.

Palloff, R.; Pratt; K. (2005). Collaborating Online: Learning Together in Community.

Rheingold, H. (1996). La comunidad virtual. Una sociedad sin fronteras. España: Editorial Gedisa

\section{Fuentes hemerográficas}

Barab, S. (2003). An Introduction to the Special Issue: Designing for Virtual Communities in the Service of Learning. The Information Society, 197-201.

Dillenbourg, P; Poirier, C. , Carles, L . (2003). Communautés virtuelles d'apprentissage: e-jargon ou nouveau paradigme? L'essor des communautés virtuelles d'apprentissage.11-48

Florido Bacallao, R.. y Florido Bacallao, M. (2003). La Educación a distancia, sus retos y posibilidades. Granada, España: Centro de Referencia para la Educación Avanzada (CREA)- Cuba. 7-27.

García Aretio, L. (1999). Fundamento y Componentes de la Educación a Distancia. Revista JIberoaméricana de Educación a Distancia. 28-39

Gutiérrez-Santiuste, E.; Gallego-Arrufat, M. (2017). Presencia Social en un Ambiente Colaborativo Virtual de Aprendizaje. Análisis de una comunidad orientada a la indagación. RMIE, 2017, Vol. (22), Núm. 75. 1169-1186 
Meirinhos, M.; Osório, A. (2009). Las Comunidades Virtuales de Aprendizaje: El Papel Central de Colaboración. Pixel-Bit. Revista de Medios y Educación, 45-60.

Ossiannilsson, E. (2012). Benchmarking ELearning in Higher Education - Lessons learned from international projects. Acta Universitatis Ouluensis - C Tec h n i c a 43 (6).

Roquet, García, G. (2006). Antecedentes históricos de la Educación a Distancia. COORDINACIÓN DE UNIVERSIDAD ABIERTA $Y$ EDUCACIÓN A DISTANCIA.8-27

\section{Fuentes electrónicas}

Barrantes Echevarría, R. (1992). Educación a Distancia. EUNED. Recuperado de http://www.educadis.com.ar/ad1.htm\#Defi niciones.

Cabero Almenara, J. (2006). Comunidades virtuales para el aprendizaje. Su utilización en la enseñanza. Edutec. Revista Electrónica de Tecnología Educativa Núm. $20 . \quad$ Recuperado de http://www.uib.es/depart/gte/gte/edutec-e/ revelec20/cabero20.htm

Carbonell, M. (2011). Abogados y Estudiantes de Derecho frente a las TIC. Recuperado de https://educacionyculturaaz.com/abogadosy-estudiantes-de-derecho-frente-a-las-tic/

Chiecher, A. ; Donolo, D. (2013). De diálogos e intercambios virtuales. La dimensión social y cognitiva de las interacciones entre alumnos. Recuperado de http:// rusc.uoc.edu/

Coll Salvador, C. (2004). Las comunidades de aprendizaje. Nuevos horizontes para la investigación y la intervención en psicología de la educación. Recuperado de http://www.ub.es/grintie/GRINTIE/Library
/public/CC_Almeria_04.pdf

Fabela, E. (2015). Técnicas de Muestreo y Prueba de Hipótesis. Recuperado de https://es.slideshare.net/ErnestoFabela1196 /u2-tecnicas-de-muestreo-y-pruebas-dehipotesis

Fernández- Sánchez Cáceres, R.; ValverdeBerrocoso, J.(2013). Comunidades de práctica: un modelo de intervención desde el aprendizaje colaborativo en entornos virtuales, España. DOI: http://dx.doi.org/10.3916/C42-2014-09.

Pazos, M.; Pérez García, A.; Salinas, J. (2001). Comunidades Virtuales: De las listas de discusión a las comunidades de aprendizaje. Recuperado de http://gte.uib.Es/articulos/cvirtuales01.pdf

Ramboll, P. (2004). Studies in the Context of the E-learning Initiative: Virtual Models of European Universities (Lot ). Draft Final Report to the EU Commission,DG Education y Culture". Recuperado de http://www.elearningeuropa.info/extras/pdf /virtual_models.pdf.

Romiszowski, A. J. (2004). "How's the Elearning Baby? Factors Leading to Success or Failure of an Educational Technology". Innovation Educational Technology 44(1). Recuperado de http://www.elearningreviews.org/topics/resourcesmanagement/project-management/2004romiszowski 